\title{
Quantum graphs where back-scattering is prohibited
}

\author{
J.M. Harrison ${ }^{1} \quad$ U. Smilansky ${ }^{2}$ \\ B. Winn ${ }^{1}$ \\ ${ }^{1}$ Department of Mathematics, Texas A $3 M$ University, College Station, Texas 77843-3368, USA. \\ ${ }^{2}$ Department of Physics of Complex Systems, Weizmann Institute of Science, Rehovot 76100, Israel.
}

$1^{\text {st }}$ August, 2007

\begin{abstract}
We describe a new class of scattering matrices for quantum graphs in which backscattering is prohibited. We discuss some properties of quantum graphs with these scattering matrices and explain the advantages and interest in their study. We also provide two methods to build the vertex scattering matrices needed for their construction.
\end{abstract}

\section{Introduction}

It has been proposed that quantised versions of metric graphs could be used to investigate the origin of spectral correlations in semi-classical quantum systems [1]. This programme has borne a considerable amount of fruit in the past decade. The culmination has been the ground-breaking work [2] providing a mechanism to understand the fidelity of spectral correlations to random matrix theory in a large class of so-called quantum graphs. It is now clear that quantum graphs can be considered at the forefront of attempts to understand the universal spectral correlations present in quantised chaotic systems [3, 4]. For a review with ample references we refer the reader to [5].

One interesting new development has been the discovery that the techniques of [1] can be used to develop trace formulæ and associated spectral zeta functions for discrete Laplacians on graphs [6]. This leads to the possibility of investigating deep connections between quantum graphs and combinatorial spectral graph theory, hitherto unexploited. In the present article we expand on some ideas introduced in [6].

We consider finite, connected graphs without loops or multiple bonds, and which have $B$ bonds and $V$ vertices. The topology of the graph is specified in terms of the $V \times V$ connectivity matrix $C$, whose $p, q$ element takes the value $1(0)$ if the vertices $p$ and $q$ are connected (not connected). We denote by $v_{j}$ the valency (degree) of the vertex $j$. A graph for which all the $v_{j}$ are equal to $v$, is called a $v$-regular graph. The metric properties are provided by the bond lengths $L_{b}, 1 \leq b \leq B$.

Spectral problems on quantum graphs are often written in terms of a $2 B \times 2 B$ unitary quantum evolution matrix $U$ defined on the vector space spanned by the directed bonds [6]. $U$ itself can be defined in terms of the $v_{j} \times v_{j}$ unitary scattering matrices $\sigma^{(j)}, j=1 \ldots V$ 
associated with each vertex of the graph.

$$
U_{(p j)(m q)}(k)=\delta_{j m} \sigma_{p q}^{(j)} \mathrm{e}^{\mathrm{i} k L_{(p j)}}
$$

where $(p j)$ is a directed bond with initial vertex $p$ and terminal vertex $j$. The spectrum under consideration is the set of values $\left\{k_{n}\right\}_{n \in \mathbb{Z}}$ of the spectral parameter $k$ for which

$$
\operatorname{det}\left[I_{2 B}-U(k)\right]=0 .
$$

(Here, and elsewhere $I_{n}$ denotes the identity matrix of dimension $n$.) A vertex scattering matrix $\sigma$ may be derived from boundary conditions of a self-adjoint operator as in [1, or specified a priori in order to provide a wider class of examples [7, 8], which is the approach we adopt here.

The classical analogue of a quantum graph is a Markovian process on the directed bonds [1] with matrix $M$ of transition probabilities related to $U$ by

$$
M_{(p j)(m q)}=\left|U_{(p j)(m q)}\right|^{2} .
$$

Thus $M$ is a doubly stochastic matrix, and hence it has dominant eigenvalue 1 . It can be interpreted as the classical operator which induces a discrete time Markovian evolution on the graph. The spectral gap $\Delta$ is the gap between the leading eigenvalue 1 and the secondlargest eigenvalue of $M$, in absolute value. It is conjectured that the rate of convergence of spectral statistics of quantum graphs to random matrix theory is governed by the rate of ergodicity in this Markov evolution. More precisely, for a sequence of graphs with $B \rightarrow \infty$ the eigenvalues of the quantum graph are given by random matrix theory if $B \Delta \rightarrow \infty[8]$.

We propose to study a new class of vertex scattering matrices defined in the following way.

Definition 1.1. A $v \times v$ unitary matrix $\sigma$ is equi-transmitting if $\sigma_{p p}=0$ for all $p$, and the off-diagonal elements have equal amplitudes; $\left|\sigma_{p q}\right|=(v-1)^{-\frac{1}{2}}$ for $p \neq q$, where $v$ is the valency of the vertex.

Our definition of an equi-transmitting matrix involves two properties which have implications for the classical analogue of the quantum graph. The first ensures that a classical particle on a directed bond has probability zero to be back-scattered to the reversal of that bond in the next time step. The second property produces democratic transmission probabilities $M_{(p j)(m q)}=\left(v_{j}-1\right)^{-1} \delta_{m j}$, for $p \neq q$

Other common choices for vertex scattering matrices are the Neumann matrix $\sigma^{[N]}$;

$$
\sigma_{p q}^{[N]}=\frac{2}{v}-\delta_{p q}
$$

and Fourier transform matrices $\sigma^{[F]}$ introduced as vertex scattering matrices in [8],

$$
\sigma_{p q}^{[F]}=\frac{1}{\sqrt{v}} \mathrm{e}^{2 \pi \mathrm{i} p q / v}
$$

Fourier transform scattering matrices allow back-scattering with equal transmission and reflection probabilities, $M_{(p j)(m q)}=v_{j}^{-1} \delta_{m j}$. The Neumann scattering matrices prefer back-scattering transitions over all other transitions put together. 
The transition probabilities induced by the three classes of scattering matrices at a vertex with a valency $v$, can be summarised by

$$
\left|\sigma_{i i}\right|^{2}=r \quad \text { and } \quad\left|\sigma_{i j}\right|^{2}=\frac{1-r}{v-1} \quad \text { for } i \neq j,
$$

where $r=(2 / v-1)^{2}$ (Neumann), $r=1 / v$ (Fourier transform) and $r=0$ (equi-transmitting).

The aims of the present manuscript can be summarised as follows:

1. To explain the motivations for studying graphs with equi-transmitting vertex scattering matrices.

2. To prove that equi-transmitting vertex scattering matrices exist for arbitrarily large $v$, and to provide practical methods to construct them.

3. To investigate their spectral properties on both the classical (gap estimates) and the quantum (spectral statistics) levels.

The next section will explain the motivation for introducing the equi-transmitting scattering matrices. While equi-transmitting matrices will be shown to have desirable qualities, an elementary calculation shows it is trivial to construct $2 \times 2$ examples, but no $3 \times 3$ equi-transmitting matrix exists. In section 3 we address the question of their existence in other dimensions, and provide several infinite sets of examples. Whether equi-transmitting matrices exist in all dimensions greater than three is an interesting open question. In section 4 we compare spectra of the matrix $M$ for graphs quantised with different scattering matrices. We find that in some fairly general situations the spectral gap in equi-transmitting quantum graphs is larger than that obtained with other scattering matrices. In section 5 we present the results of numerical simulations using equitransmitting quantum graphs that show that random matrix statistics are reproduced.

\section{Motivations}

In this section we shall try to explain the motivations for the introduction of the equitransmitting vertex matrices.

A fundamental building block in the spectral theory for quantum graphs and a key tool in understanding spectral correlations [5], is the trace formula [1, 9]. It relates the quantum spectrum of a graph, $\left\{k_{n}\right\}_{n \in \mathbb{Z}}$, to the length spectrum of its periodic orbits, expressed as an identity of distributions:

$$
\sum_{n \in \mathbb{Z}} \mathrm{e}^{\mathrm{i} k_{n} u}=\mathscr{L} \delta(u)+\sum_{\text {p.o. }} \frac{\ell_{p}}{r_{p}}\left(A_{p} \delta\left(u-\ell_{p}\right)+\bar{A}_{p} \delta\left(u+\ell_{p}\right)\right) .
$$

The first term on the right-hand side of $(2.6)$ is the Weyl term. $\mathscr{L}$ denotes twice the total length of the graph, and plays the rôle of the volume. The more interesting second term is a sum which goes over classes of closed itineraries on the graph equivalent up to cyclic permutations of the edges. We call these periodic orbits of the graph. The metric length of an orbit is denoted $\ell_{p}$, and $r_{p}$ is the number of times the orbit is a repetition of a shorter one. The amplitude factor $A_{p}$ is the product of all elements of the vertex scattering matrices encountered as the orbit passes from one bond to the next. 
Since equi-transmitting matrices forbid back-scattering transitions, any orbit in which a traversal of a bond is followed immediately by the traversal of its reverse is eradicated from the sum. This significantly reduces the number of orbits which need to be considered - in fact the orbits that remain are exactly the closed geodesics considered in combinatorial graph theory which will be discussed below. For a $v$-regular graph the asymptotic number of orbits of period $n$ is reduced from $v^{n} / n$ to $(v-1)^{n} / n$.

We anticipate that using equi-transmitting scattering matrices will significantly simplify periodic orbit theories in quantum graphs, and perhaps lead to new interesting problems and breakthroughs. The following will serve as an example: A theorem of Gutkin and Smilansky [10] guarantees that one can "hear" the shape of a graph if the bond lengths are rationally independent and if the vertex scattering matrices are "properly connecting". The equi-transmitting scattering matrices do not belong to the latter class, and the question if one can "hear" equi-transmitting graphs is open. The proof of the above mentioned theorem is based on the trace formula, and relies heavily on the special properties of the 2-periodic orbits which are absent from the trace formula of equi-transmitting graphs. Thus, a completely novel approach has to be developed.

The study of quantum graphs with equi-transmitting vertex scattering matrices leads to connections with objects that have been extensively studied in combinatorial graph theory, such as the Ihara-Selberg zeta function [11] and its generalisations. One way to see this connection is by studying the spectrum of the classical evolution operation $M$, which is defined as the zero set of the secular function

$$
Z_{M}(\mu):=\operatorname{det}\left[I_{2 B}-\mu M\right] \text {. }
$$

This equation can be used to derive a classical (Rouelle like) trace formula which is based on the periodic orbits on the graph weighted by the products of scattering probabilities along the orbit. When dealing with equi-transmitting vertex scattering matrices, the trace formula (and the corresponding zeta function) includes only periodic orbits without back-scatter. These are the orbits which appear in the Ihara-Selberg zeta function and its extensions [12, 13, 14, 15]. Moreover, the zeta functions are related by simple transformations, so that an interesting correspondence between the two seemingly unrelated problems can be established. We have mentioned previously the classical spectral gap $\Delta$, and its conjectured influence on the spectral statistics in the corresponding quantum graph. Establishing bounds on $\Delta$ arises in the study of the Ihara-Selberg zeta function, which makes an intriguing link between spectral statistics and number theory. (See [16] for a review of the connections between arithmetical zeta functions and spectral statistics.) We shall make use of these connections here (see section 4).

We are not able to prove that graphs with equi-transmitting vertex scattering matrices display spectral statistics which reproduce the predictions of random matrix theory. We present, however, quite convincing numerical evidence showing that both their spectral repulsion and spectral rigidity adhere to the predictions of the canonical random matrix ensembles. Whether this observation is valid and can be rigorously formulated is an open problem awaiting future research. 


\section{Existence and construction}

In this section we shall show that the set of equi-transmitting matrices is not empty or trivial. It is easy to construct equi-transmitting matrices in dimension 2. It is equally easy to show that equi-transmitting matrices do not exist in dimension 3 . We are not able to provide a list of the dimensions for which equi-transmitting matrices exist. We can, however, show that this list is infinite. We do it by constructing examples of equitransmitting matrices using skew-Hadamard matrices [17, 18, 19], and Dirichlet characters [20].

\subsection{Construction of equi-transmitting matrices using Hadamard matrices}

Definition 3.1. A Hadamard matrix $H$ is a matrix whose entries are \pm 1 , and whose columns are orthogonal. A skew-Hadamard matrix is a Hadamard matrix satisfying the additional condition

$$
H+H^{\mathrm{T}}=2 I_{v}
$$

Proposition 3.2. Let $H$ be a $v \times v$ skew-Hadamard matrix. Then

$$
\sigma=\frac{1}{\sqrt{v-1}}\left(H-I_{v}\right)
$$

is an equi-transmitting matrix.

Proof. All entries of $\sigma$ are $\pm(v-1)^{-1 / 2}$ except for the zero entries along the diagonal. So we need only check unitarity. But this is clear, since

$$
\begin{aligned}
\sigma \sigma^{\mathrm{T}} & =\frac{1}{v-1}\left(H H^{\mathrm{T}}-\left(H+H^{\mathrm{T}}\right)+I_{v}\right) \\
& =I_{v}
\end{aligned}
$$

since necessarily $H H^{\mathrm{T}}=v I_{v}$.

Hadamard matrices have been conjectured to exist in dimensions 1,2 and all multiples of 4. This conjecture appears to date back to [21]. Various constructions of skewHadamard matrices are known [17, 18, 19]. Currently there are constructions of skewHadamard matrices for all dimensions which are a multiple of 4 up to and including 184 [22, 23], plus other infinite sets of dimensions.

\subsection{Construction of equi-transmitting matrices using Dirichlet characters}

This method provides equi-transmitting matrices of dimensions $P+1$ where $P$ is prime. 
Proposition 3.3. Let $P$ be an odd prime and let $\chi$ be a non-trivial Dirichlet character modulo $P$. Let $C$ be the $P \times P$ matrix defined by $C_{j \ell}=\chi(\ell-j)$. Then

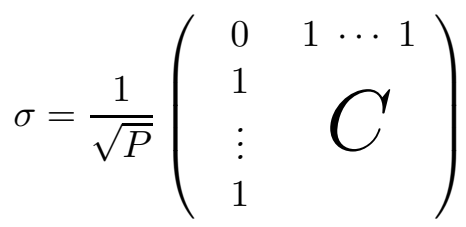

is equi-transmitting.

We first need an auxiliary lemma.

Lemma 3.4. Let $P$ be an odd prime, $\chi$ a non-trivial Dirichlet character, and $j \in \mathbb{Z}$. Then

$$
\sum_{n=0}^{P-1} \chi(n-j) \overline{\chi(n)}=\left\{\begin{aligned}
P-1 & \text { if } j \equiv 0 \quad \bmod P, \\
-1 & \text { otherwise. }
\end{aligned}\right.
$$

Proof. If $j \equiv 0$ modulo $P$ then

$$
\sum_{n=0}^{P-1}|\chi(n)|^{2}=\sum_{n=1}^{P-1} 1=P-1 .
$$

Otherwise, writing $n^{-1}$ for the multiplicative inverse of $n$ in the finite field $\mathbb{Z} / P \mathbb{Z}$,

$$
\begin{aligned}
\sum_{n=0}^{P-1} \chi(n-j) \overline{\chi(n)} & =\sum_{n=1}^{P-1} \chi(n-j) \chi(n)^{-1} \\
& =\sum_{n=1}^{P-1} \chi\left((n-j) n^{-1}\right) \\
& =\sum_{n=1}^{P-1} \chi\left(1-j n^{-1}\right)
\end{aligned}
$$

Now since $n^{-1}$ runs over all invertible elements in the field, the only argument which does not appear in the sum is 1 . So

$$
\begin{aligned}
\sum_{n=0}^{P-1} \chi(n-j) \overline{\chi(n)} & =\sum_{m=0}^{P-1} \chi(m)-\chi(1) \\
& =-1
\end{aligned}
$$

Using orthogonality of Dirichlet characters [20] and the known value $\chi(1)=1$.

Proof of proposition 3.3. We first observe that $|\chi(n)|=1$ unless $n \equiv 0$ whence $\chi(0)=0$, so $\sigma$ has the required form. It is easy to see that unitarity will follow once we prove that

$$
C C^{\dagger}=\left(\begin{array}{cccc}
P-1 & -1 & \cdots & -1 \\
-1 & P-1 & \cdots & -1 \\
\vdots & \vdots & \ddots & \vdots \\
-1 & -1 & \cdots & P-1
\end{array}\right)
$$


For this, note that the inner product of the $j^{\text {th }}$ and $\ell^{\text {th }}$ columns of $C$ can be written

$$
\begin{aligned}
\sum_{m=1}^{P} C_{m j} \overline{C_{m \ell}} & =\sum_{m=1}^{P} \chi(j-m) \overline{\chi(\ell-m)} \\
& =\sum_{n=0}^{P-1} \chi(n+j-\ell) \overline{\chi(n)},
\end{aligned}
$$

via a change of index of summation. Now (3.15) follows by using the lemma to evaluate the sum (3.16).

Corollary 3.5. Let $P$ be a prime congruent to 1 modulo 4 . Then there exists a symmetric equi-transmitting matrix of dimension $P+1$.

Proof. We use the construction in proposition 3.3 with the Legendre symbol as the Dirichlet character $\chi(n)=\left(\frac{n}{P}\right)$ where

$$
\left(\frac{n}{P}\right)=\left\{\begin{aligned}
0 & \text { if } n \equiv 0 \bmod P, \\
1 & \text { if } n \text { is a square modulo } P, \\
-1 & \text { if } n \text { is not a square modulo } P .
\end{aligned}\right.
$$

To show that $\sigma$ is symmetric it suffices to show that the circulant matrix $C$ is symmetric. To see this, note that

$$
\chi(P-m)=\left(\frac{m(-1)}{P}\right)=\left(\frac{m}{P}\right)\left(\frac{-1}{P}\right)=\left(\frac{m}{P}\right)=\chi(m)
$$

since $(-1 / P)=1$ if $P \equiv 1$ modulo 4 (Euler).

By Dirichlet's theorem, corollary 3.5 provides infinitely many examples of symmetric equi-transmitting matrices.

The constructions in propositions 3.2 and 3.3 give many examples of equi-transmitting matrices of even dimensions. We can construct an example of an equi-transmitting matrix in dimension 5 ,

$$
\sigma=\frac{1}{2}\left(\begin{array}{ccccc}
0 & 1 & 1 & 1 & 1 \\
1 & 0 & 1 & \omega & \omega^{2} \\
1 & 1 & 0 & \omega^{2} & \omega \\
1 & \omega & \omega^{2} & 0 & 1 \\
1 & \omega^{2} & \omega & 1 & 0
\end{array}\right), \quad \text { where } \omega=\mathrm{e}^{2 \pi \mathrm{i} / 3}
$$

However, apart from this example we do not have any examples in odd dimensions. These equi-transmitting matrices do not appear to have been studied in the literature. An examination of (3.18) might lead one to suspect that such matrices can be constructed for dimension $v$ by using entries that are $(v-2)^{\text {th }}$ roots of unity. However we have exhaustively checked for the case $v=7$ and shown this to be false.

\section{Properties of graphs with equi-transmitting scattering matrices}

Now that we have demonstrated the existence of equi-transmitting matrices, we can study the quantum and classical evolutions which they induce. In particular, we shall use some 
results from combinatorial graph theory to demonstrate the advantages gained by studying graphs with equi-transmitting vertex scattering matrices.

An important tool in the preceding discussion is the spectrum of the connectivity matrix $C$ defined in the introduction. Since $C$ is symmetric its eigenvalues $\mu_{j}$ are real, and we order them: $\mu_{V-1} \leqslant \cdots \leqslant \mu_{1} \leqslant \mu_{0}$.

We shall consider $v$-regular graphs and to avoid trivial cases we will assume throughout that $v>3$. A connected $v$-regular graph has the property that its connectivity matrix has largest eigenvalue $\mu_{0}=v$ and it is simple. A $v$-regular graph is called Ramanujan if all other eigenvalues of $C$ are contained in the interval $[-2 \sqrt{v-1}, 2 \sqrt{v-1}]$. Ramanujan graphs are of interest in computer science and communication network theory since they are sparse yet highly connected [24, 25].

We shall discuss the spectrum of the classical evolution operator $M$ on a $v$-regular graph constructed by using vertex scattering matrices of the types listed in (1.5). We shall relate them to the spectrum of the connectivity matrix $C$ by the following theorem.

Theorem 4.1. Let $M$ be the doubly stochastic transition probabilities matrix associated to a quantum $v$-regular graph with unitary vertex scattering matrices $\sigma$ which satisfy (1.5) for some $r>0$. Let the eigenvalues of $C$ be $\mu_{0} \geqslant \mu_{1} \geqslant \cdots \geqslant \mu_{V-1}$. Let

$$
u_{j}:=\frac{(1-r) \mu_{j}+\sqrt{(1-r)^{2} \mu_{j}^{2}-4(1-r v)(v-1)}}{2(v-1)}
$$

and

$$
\tilde{u}_{j}:=\frac{(1-r) \mu_{j}-\sqrt{(1-r)^{2} \mu_{j}^{2}-4(1-r v)(v-1)}}{2(v-1)} .
$$

The spectrum of $M$ consists of the points

$$
u_{0}, \ldots, u_{V-1}, \tilde{u}_{0}, \ldots, \tilde{u}_{V-1}, \frac{|1-r v|}{v-1},-\frac{|1-r v|}{v-1}
$$

where the last two points are listed with multiplicity $\frac{(v-2) V}{2}$.

Proof. Let $W$ be the matrix representing Hashimoto's bond (edge) adjacency operator [26, 15] defined as:

$$
W_{(p j)(m q)}=\delta_{m j}\left(1-\delta_{p q}\right) .
$$

It has entries equal to 1 only when two directed bonds follow each other at a common vertex $j$, but excluding back-scattering. The form of the vertex scattering matrices $\sigma$ of interest here is provided by (1.5). It implies that for $v$-regular graphs,

$$
M=\left(\frac{1-r}{v-1}\right) W+r J
$$

where $r=(2 / v-1)^{2}$ (Neumann), $r=1 / v$ (Fourier transform) and $r=0$ (equi-transmitting). The characteristic polynomial of $M$, $\operatorname{det}\left[u I_{2 B}-M\right]$ is related to a graph theoretic zeta function developed in [27]. Bartholi's theorem [27, 28] implies an equivalent form for the characteristic polynomial in terms of the matrix $C$,

$$
\operatorname{det}\left[u I_{2 B}-M\right]=\left(u^{2}-\frac{(1-r v)^{2}}{(v-1)^{2}}\right)^{(v-2) V / 2} \operatorname{det}\left[\left(u^{2}+\frac{1-r v}{v-1}\right) I_{V}-\frac{1-r}{v-1} C u\right] .
$$


It follows that eigenvalues of $M$ are solutions to

$$
u^{2}-\frac{(1-r v)^{2}}{(v-1)^{2}}=0
$$

(with multiplicity $(v-2) V / 2)$ and

$$
u^{2}-\frac{1-r}{v-1} \mu_{j} u+\frac{1-r v}{v-1}=0,
$$

for $j=0, \ldots, V-1$.

For regular graphs with equi-transmitting scattering matrices, theorem 4.1 shows that the eigenvalues of $M$ are (up to a scaling) at the positions of the poles of the Ihara-Selberg zeta function [11] of the graph, as was noted in [6]. The proof in this case follows from Bass' identity [29] of which Bartholi's theorem is a generalisation.

Theorem 4.1 will enable us to compare equi-transmitting scattering matrices with others of type (1.5) (see Theorem 4.3 below). It also has a number of other consequences which may be of independent interest.

Corollary 4.2. $\quad$ - If $r \geqslant 1 / v$ then all eigenvalues of $M$ are real.

- If $r=1 / v$ (e.g. Fourier transform scattering matrix) then the eigenvalues of $M$ are

$$
\frac{\mu_{0}}{v}, \ldots, \frac{\mu_{V-1}}{v}, 0
$$

and the 0 has multiplicity $(v-1) V$.

- If $r<1 / v$ then $u_{j}$ and $\tilde{u}_{j}$ are real iff $\left|\mu_{j}\right| \geqslant \frac{2}{1-r} \sqrt{(1-r v)(v-1)}$.

- If $r<1 / v$ and $\left|\mu_{j}\right|<\frac{2}{1-r} \sqrt{(1-r v)(v-1)}$ then

$$
\left|u_{j}\right|=\left|\tilde{u}_{j}\right|=\sqrt{\frac{1-r v}{v-1}}
$$

In particular, for a $v$-regular graph which is Ramanujan, and has equi-transmitting scattering matrices, all but the eigenvalue 1 lie in a disc of radius $(v-1)^{-1 / 2}$ about the origin.

Theorem 4.3. Consider a v-regular graph. Let the spectral gap for the quantum graph with equi-transmitting scattering matrices be $\Delta_{\text {et }}$ and denote by $\Delta_{r}$ the spectral gap for the same graph with scattering matrices $\sigma$ satisfying (1.5) for some $r>0$. Then there exists $\varepsilon>0$ such that if $C$ has an eigenvalue in either of the intervals $(2 \sqrt{v-1}-\varepsilon, v)$ or $[-v,-2 \sqrt{v-1}+\varepsilon)$ then $\Delta_{\text {et }}>\Delta_{r}$.

We give the proof of theorem 4.3 at the end of this section. Theorem 4.3 demonstrates that in some fairly general situations the spectral gap arising in quantum graphs with equi-transmitting scattering matrices is larger than the spectral gap with other kinds of scattering matrices. Notice that if one considers a sequence of $v$-regular graphs with $V \rightarrow \infty$ the Alon-Boppana bound [24, Theorem 1.3.1] states that

$$
\liminf _{V \rightarrow \infty} \mu_{1} \geqslant 2 \sqrt{v-1}
$$


In other words, theorem 4.3 will apply eventually.

In the interests of full disclosure we point out that for some other families of graphs equitransmitting matrices will not necessarily lead to a larger (although still large) spectral gap, e.g. fully-connected graphs.

Before giving the proof, we provide one more lemma.

Lemma 4.4. Let $0 \leqslant \mu<v$. Then define

$$
f_{\mu}(r):=\frac{(1-r) \mu+\sqrt{(1-r)^{2} \mu^{2}+4(v r-1)(v-1)}}{2(v-1)} .
$$

Then $f_{\mu}(r)$ is real and strictly increasing on $1-\frac{2(v-1)}{\mu^{2}}\left(v-\sqrt{v^{2}-\mu^{2}}\right) \leqslant r \leqslant 1$.

Proof. It is convenient to define a new variable $X=v(1-r) /(v-1)$, so that $f_{\mu}$ becomes, after some manipulation,

$$
f_{\mu}(X)=\frac{1}{2 v}\left(X \mu+\sqrt{\mu^{2} X^{2}+4 v^{2}(1-X)}\right),
$$

and is real if $0 \leqslant X \leqslant \frac{2 v^{2}-2 v \sqrt{v^{2}-\mu^{2}}}{\mu^{2}}$. Differentiating,

$$
\begin{aligned}
\frac{\mathrm{d} f_{\mu}}{\mathrm{d} X} & =\frac{1}{2 v}\left(\mu-\frac{2 v^{2}-\mu^{2} X}{\sqrt{\mu^{2} X^{2}+4 v^{2}(1-X)}}\right) \\
& \leqslant \frac{1}{2 v}\left(\mu-\frac{(2-X) v^{2}}{\sqrt{v^{2}(X-2)^{2}}}\right) \\
& =\frac{1}{2 v}(\mu-v)<0
\end{aligned}
$$

since $\sqrt{(X-2)^{2}}=2-X$, as $0 \leqslant X<2$. As $\frac{\mathrm{d} X}{\mathrm{~d} r}<0$ it follows that $f_{\mu}(r)$ is strictly increasing.

Proof of theorem 4.3. We assume $\left|\mu_{1}\right| \geqslant\left|\mu_{V-1}\right|$. If this is not the case then the argument below holds mutatis mutandis replacing $\mu_{1}$ by $\mu_{V-1}$.

If the graph is Ramanujan, and if $r<1 / v$, then choose $\varepsilon$ so that

$$
\frac{2-r v}{1-r} \sqrt{v-1}<2 \sqrt{v-1}-\varepsilon
$$

Since $v>3$ if $r>0$ such an $\varepsilon$ can always be found.

As $\mu_{1}>\frac{2-r v}{1-r} \sqrt{v-1}>\frac{2}{1-r} \sqrt{(1-r v)(v-1)}$ by corollary $4.2 u_{1}=f_{\mu_{1}}(r)$ is real and $\Delta_{r}=1-u_{1}$, where

$$
u_{1}=\frac{(1-r) \mu_{1}+\sqrt{(1-r)^{2} \mu_{1}^{2}-4(1-r v)(v-1)}}{2(v-1)}>\frac{1}{\sqrt{v-1}} .
$$

For Ramanujan graphs, $\Delta_{\text {et }}=1-\frac{1}{\sqrt{v-1}}>\Delta_{r}$. 
If the graph is Ramanujan and $r \geqslant 1 / v$ then choose $\varepsilon$ to satisfy

$$
\frac{v}{\sqrt{v-1}}<2 \sqrt{v-1}-\varepsilon
$$

By lemma 4.4,

$$
u_{1} \geqslant f_{\mu_{1}}(1 / v)=\frac{\mu_{1}}{v}>\frac{1}{\sqrt{v-1}}
$$

and again we have $\Delta_{\text {et }}>\Delta_{r}$.

If the graph is not Ramanujan, then $u_{1}$ is still real since

$$
\mu_{1}>2 \sqrt{v-1}>\frac{2}{r-1} \sqrt{(1-r v)(v-1)} \quad \text { if } r<1 / v
$$

so by lemma 4.4

$$
1-\Delta_{r}=f_{\mu_{1}}(r)>f_{\mu_{1}}(0)=1-\Delta_{\mathrm{et}}
$$

\section{$5 \quad$ Numerical simulations}

The results of the previous section show that well-connected quantum graphs with equitransmitting scattering matrices can have large spectral gaps. According to the Tanner conjecture [8] the spectral statistics should converge to the statistics of ensembles of random matrix theory. To illustrate this we present the results of some numerical calculations of the nearest-neighbour spacing density $P(s)$ for points in the spectrum of a quantum graph, and the variance $V(L)$ of the number of points in an interval of length $L$.

To calculate these spectral statistics we did not solve (1.2) directly. Rather we took an approach which is known to be equivalent. We replaced the phases $k L_{(p j)}$ in (1.1) by random phases in the interval $[0,2 \pi]$ and diagonalised the resulting matrices. The statistics of the re-scaled eigen-phases approach those of the spectrum defined by (1.2) in the limit as $B \rightarrow \infty$ if the bond lengths are not rationally related, and drawn from a narrowing interval as $B \rightarrow \infty$ [5, 30].
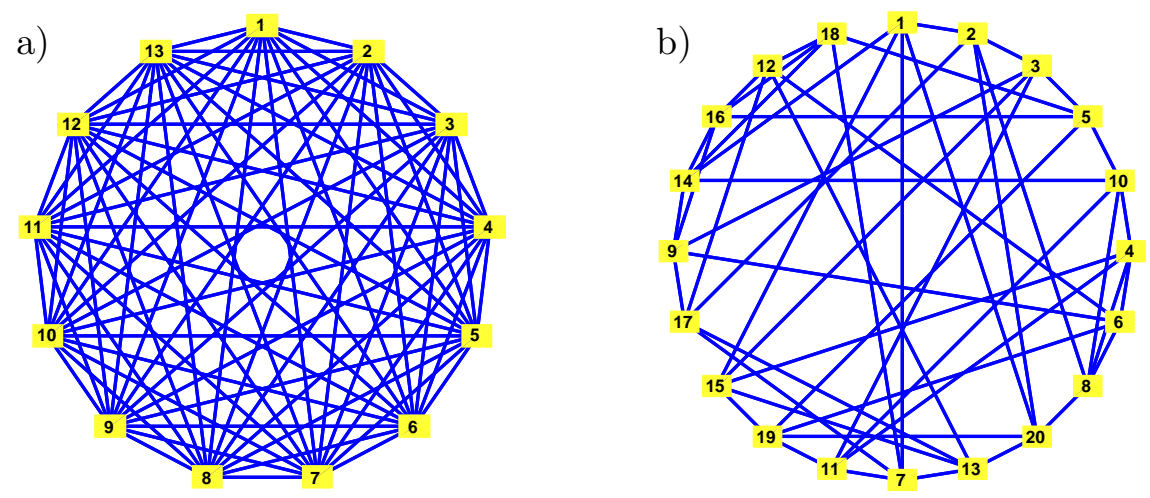

Figure 1: The graphs used in the numerical simulations: a) A complete graph with 13 vertices, b) a 5-regular graph with 20 vertices. 
In figure 2 we plot the nearest neighbour distribution and number variance for the 5 -regular graph in figure 1b) with the equi-transmitting vertex scattering matrix given explicitly in (3.18). Since $\sigma$ is symmetric we expect the spectral statistics to approach those of the Gaussian orthogonal ensemble (GOE) of random matrices. We also plot in figure 2 the corresponding limiting curves for Gaussian orthogonal ensemble and Gaussian unitary ensemble (GUE) [31. We see, as expected, agreement to the Gaussian orthogonal ensemble curves, even for such a relatively small graph.

In figure 3 we present the corresponding numerics for the complete graph on 13 vertices (figure 1a) with an anti-symmetric equi-transmitting scattering matrix at each vertex. In this case we expect convergence to the Gaussian unitary ensemble statistics, and this is also clearly demonstrated in the figure.
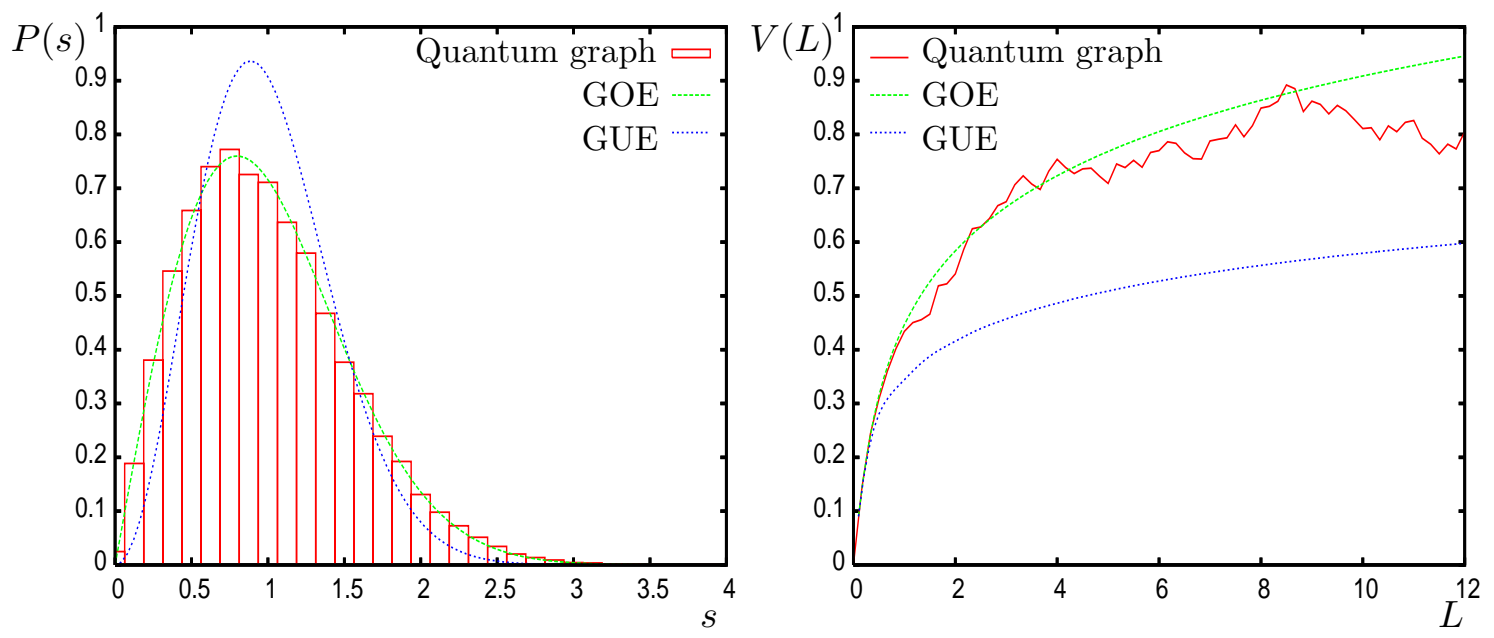

Figure 2: Spectral statistics for a quantum regular graph with symmetric scattering matrices. On the left is a plot of the nearest-neighbour density; on the right is the number variance.

Given the utility of these equi-transmitting matrices, we believe the study of their existence in dimensions for which we do not currently have examples is interesting and merits further investigation.

\section{Acknowledgements}

We are grateful for interesting discussions with Gregory Berkolaiko and Nicole Raulf.

The work of JMH and BW is supported by the National Sciences Foundation under research grant DMS-0604859. US acknowledges support from the Minerva Center for nonlinear Physics, the Einstein (Minerva) Center at the Weizmann Institute and BSF grant 2006065 .

Part of this work was carried out while the authors were visiting the Isaac Newton Institute for Mathematical Sciences, Cambridge, UK. US acknowledge the EPSRC grant 531174 which supported his stay. The stay of JMH and BW was partially supported by National Sciences Foundation grant DMS-0648786. 

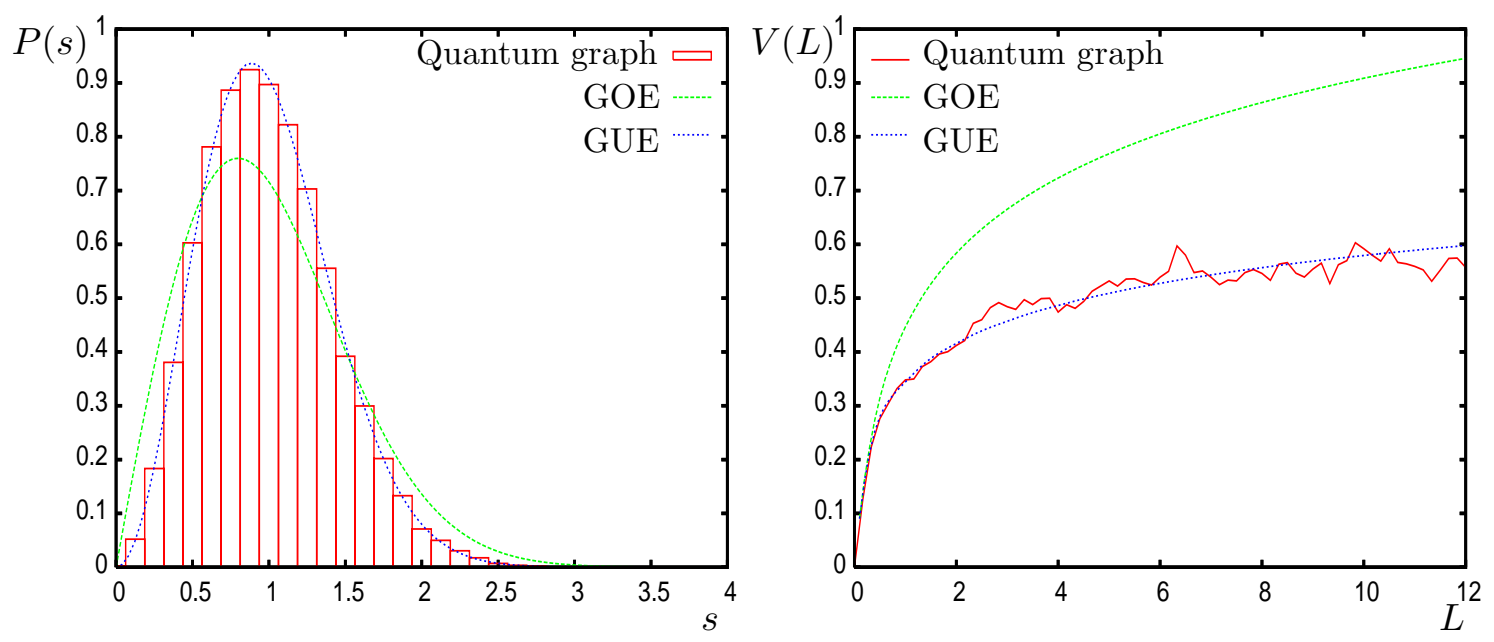

Figure 3: Spectral statistics for the quantum complete graph with anti-symmetric scattering matrices. On the left is a plot of the nearest-neighbour density; on the right is the number variance.

\section{References}

[1] T. Kottos and U. Smilansky (1999) "Periodic orbit theory and spectral statistics for quantum graphs," Ann. Phys. 274, pp. 76-124.

[2] S. Gnutzmann and A. Altland (2004) "Universal spectral statistics in quantum graphs," Phys. Rev. Lett. 93, art. no. 194101.

[3] O. Bohigas, M.-J. Giannoni, and C. Schmit (1984) "Characterization of chaotic quantum spectra and universality of level fluctuation laws," Phys. Rev. Lett. 52, pp. 1-4.

[4] G. Casati, F. Valz-Griz, and I. Guarneri (1980) "On the connection between the quantization of nonintegrable systems and statistical theory of spectra," Lett. Nuovo Cimento 28, pp. 279-282.

[5] S. Gnutzmann and U. Smilansky (2006) "Quantum graphs: Applications to quantum chaos and universal spectral statistics," Adv. Phys. 55, pp. 527-625.

[6] U. Smilansky (2007) "Quantum chaos on discrete graphs," J. Phys. A 40, pp. F621F630.

[7] H. Schanz and U. Smilansky (2000) "Spectral statistics for quantum graphs: periodic orbits and combinatorics," Phil. Mag. B 80, pp. 1999-2021. (Proceedings of the Australian summer school on quantum chaos and mesoscopics).

[8] G. Tanner (2001) "Unitary stochastic matrix ensembles and spectral statistics," $J$. Phys. A 34, pp. 8485-8500.

[9] J. P. Roth "Le spectre du Laplacien sur un graphe," in Theorie du potentiel (A. Dold and B. Eckmann, eds.) Lecture notes in mathematics pp. 521-539 Springer-Verlag 1983. In French. 
[10] B. Gutkin and U. Smilansky (2001) "Can one hear the shape of a graph?" J. Phys. A 31, pp. 6061-6068.

[11] Y. Ihara (1966) "On discrete subgroups of the two by two projective linear group over p-adic fields," J. Math. Soc. Japan 18, pp. 219-235.

[12] H. M. Stark and A. A. Terras (1996) "Zeta functions of finite graphs and coverings," Adv. Math. 121, pp. 124-165.

[13] H. M. Stark "Multipath zeta functions of graphs," in Emerging applications of number theory (Minneapolis, MN, 1996) vol. 109 of IMA Vol. Math. Appl. pp. 601-615 New York: Springer 1999.

[14] M. Kotani and T. Sunada (2000) "Zeta functions of finite graphs," J. Math. Sci. Univ. Tokyo 7, pp. 7-25.

[15] M. D. Horton, H. M. Stark, and A. A. Terras "What are zeta functions of graphs and what are they good for?" in Quantum graphs and their applications (G. Berkolaiko, R. Carlson, S. Fulling, and P. Kuchment, eds.) no. 415 in Contemporary Mathematics pp. 173-189 AMS 2006. Proceedings of Joint Summer Research Conference, Snowbird, Utah.

[16] J. P. Keating and N. C. Snaith (2003) "Random matrices and L-functions," J. Phys. A 36, pp. 2859-2881.

[17] J. Williamson (1944) "Hadamard's determinant theorem and the sum of four squares," Duke Math. J. 11, pp. 65-81.

[18] E. C. Johnsen (1966) "Integral solution to the incidence equation for finite projective plane cases of orders $n \equiv 2 \bmod 4 ., "$ Pacific J. Math. 17, pp. 97-120.

[19] A. L. Whiteman (1971) "An infinite family of skew Hadamard matrices," Pacific J. Math. 38, pp. 817-822.

[20] K. Ireland and M. Rosen A classical introduction to modern number theory vol. 84 of Graduate Texts in Mathematics. New York: Springer-Verlag second ed. 1990.

[21] R. E. A. C. Paley (1933) "On orthogonal matrices," J. Math and Phys. 12, pp. 311320. Journal now called Studies in Applied Mathematics.

[22] D. Ž. Đoković (1992) "Skew Hadamard matrices of order $4 \times 37$ and $4 \times 43$.," J. Combinatorial Theory 61, pp. 319-321.

[23] S. Georgiou, C. Koukouvinos, and S. Stylianou (2002) "On good matrices, skew Hadamard matrices and optimal designs," Comput. Statist. Data Anal. 41, pp. 171184.

[24] G. Davidoff, P. Sarnak, and A. Valette Elementary Number Theory, Group Theory, and Ramanujan Graphs. No. 55 in London Mathematical Society Student Texts Cambridge 2003.

[25] M. R. Murty (2003) "Ramanujan graphs," J. Ramanujan Math. Soc. 18, pp. 1-20. 
[26] K. Hashimoto "Zeta functions of finite graphs and representations of $p$-adic groups," in Automorphic forms and geometry of arithmetic varieties vol. 15 of Adv. Stud. Pure Math. pp. 211-280 Boston, MA: Academic Press 1989.

[27] L. Bartholdi (1999) "Counting paths in graphs," Enseign. Math. (2) 45, pp. 83-131.

[28] H. Mizuno and I. Sato (2005) "A new proof of Bartholdi's theorem," J. Algebraic Combin. 22, pp. 259-271.

[29] H. Bass (1992) "The Ihara-Selberg zeta function of a tree lattice," Internat. J. Math. 3, pp. 717-797.

[30] G. Berkolaiko and B. Winn. In preparation.

[31] M. L. Mehta Random Matrices. Academic Press 1991. 